\title{
Teager Energy Based Filter-Bank Cepstra in EEG Classification for Seizure Detection Using Radial Basis Function Neural Network
}

\author{
Chandrakar Kamath \\ Electronics and Communication Department, Manipal Institute of Technology, Manipal 576104, India
}

Correspondence should be addressed to Chandrakar Kamath; chandrakar.kamath@gmail.com

Received 2 September 2013; Accepted 2 October 2013

Academic Editors: R. Grebe and V. Krajca

Copyright (C) 2013 Chandrakar Kamath. This is an open access article distributed under the Creative Commons Attribution License, which permits unrestricted use, distribution, and reproduction in any medium, provided the original work is properly cited.

\begin{abstract}
About $1-3 \%$ of the world population suffers from epilepsy. Epileptic seizures are abnormal sudden discharges in the brain with signatures manifesting in the electroencephalograph (EEG) recordings by frequency changes and increased amplitudes. These changes, in this work, are captured through static and dynamic features derived from three Teager energy based filter-bank cepstra (TE-FB-CEPs). We compared the performance of linear, logarithmic, and Mel frequency scale TE-FB-CEPs using radial basis function neural network in general epileptic seizure detection. The comparison is tried on eight different classification problems which encompass all the possible discriminations in the medical field related to epilepsy. In a previous study, using traditional cepstrum on the same database, we had found that the composite vectors showed a degraded performance in seizure detection. In this study, however, irrespective of frequency scaling used, it is found that the composite vectors of TE-FB-CEPs maintain excellent overall accuracy in all the eight classification problems.
\end{abstract}

\section{Introduction}

Epilepsy is a chronic neurological disorder with a prevalence of $1-3 \%$ of the world population [1]. Epilepsy is characterized by recurrent unprovoked epileptic seizures, which are episodic and rapidly evolving temporary events. The seizures reflect the clinical signs of an excessive and hypersynchronous activity of the neurons in the brain. The symptoms during seizure vary depending on the location and extent of the affected brain tissue. The unforeseen nature of these seizures make the daily life of patients miserable with temporary impairments of perception, speech, memory, motor control, and/or consciousness and sometimes may lead to enhanced risk of injury and/or death. Epilepsy can be controlled but not cured with antiepileptic medication. The epileptic brain can be considered to function in one of the two states: interictal state with occasional transient waveforms, as isolated spikes, sharp waves, or spike-wave complexes and ictal (seizure) state with continuous discharge of polymorphic waveforms of varying amplitude and frequency, spike and sharp wave complexes, rhythmic hypersynchrony, or electrocerebral inactivity observed over a duration longer than average duration of these abnormalities during interictal intervals [2]. Until now, not much is understood about the occurrence and mechanism underlying the epileptic seizure. Long-term inpatient/ambulatory electroencephalograph (EEG), lasting from a few hours to several days which definitely contain interictal and ictal hallmark of epilepsy, is required clinically to diagnose, monitor, and localize the epileptogenic zone [3]. The EEG during seizure is significantly different from that of the interictal state and that of a normal subject. The traditional methods rely on welltrained clinical neurophysiologists who visually inspect the entire lengthy EEG signals, which not only are tedious and time-consuming but cost higher. Therefore, many automated epileptic detection systems have been developed using different approaches in the recent years [4]. Such automated systems reduce the time taken to review offline the long-term EEG recordings significantly and facilitate the neurologist to diagnose and treat more patients in a given time. This implies that the selected feature set must be such that besides accuracy in seizure detection, the processing time must be 
very short. However, the wide variety of EEG patterns that characterize the nature of seizures, such as spikes and waves, low-amplitude desynchronization, polyspike activity, rhythmic waves for a wide range of frequencies, and amplitudes, tend to increase the complexity of the automated seizure detection problem.

Selecting an optimal set of significant features plays an important role in developing a good classification system, particularly when using pattern recognition paradigm. A general thumb rule is to use those features which capture those aspects of the time series which are relevant for discriminating between the classes. To meet higher accuracy it is not adequate if we have the best pattern classification system. It is found that performance of majority of the classifiers deteriorates when some of the selected features are redundant. Thus, it is important that the selected features must be screened for redundancy and irrelevancy. Also the number of extracted features must be small. Otherwise it will add onto computational overheads and a longer processing time. Different methods have been used to extract diverse features, including those which capture frequency, energy, and structural content of the signal, for the task of epileptic seizure detection [5-8]. Epileptic seizure analysis can be divided into three categories: (1) epileptic seizure detection, (2) epileptic seizure prediction, and (3) epileptic seizure origin localization [9]. The epileptic seizure detection methods, usually, aim to detect patterns in EEG recordings that are a manifestation of an epileptic seizure. The entire procedure of methods developed for automated epileptic seizure detection can be subdivided into two stages, namely, (i) feature extraction and (ii) classification [10]. We have adopted this approach to epilepsy detection. In a recent study, we had found that the overall performance of both the composite vectors of the traditional cepstrum (CEP) deteriorates compared to that of the baseline vector in the seizure detection and classification of EEG segments [11]. However, there are not many studies who have explored to a sufficient depth other features used in different domains of signal processing, for example, the occasionally used feature such as cepstrum based on Teager energy operator, being tried for seizure detection. Teager energy operator based cepstral features (TEOCEP, TECC) [12-14] and Teager energy based Mel cepstral coefficients (T-MFCC) [15] have been used for speech recognition and analysis. TEOCEP and TECC composite cepstral vectors too have been found to add to improved performance in speech processing $[12,14]$. However, to the best of our knowledge, this is the first study where Teager energy based filter-bank cepstra (TE-FB-CEPs) are applied and investigated for unbalanced general EEG data classification. Also, no other work addresses all the eight classification problems discussed below, which encompass all the possible discriminations in the medical field related to epilepsy. This is a pilot study primarily intended to (1) study the effect of TE-FB-CEP on the EEG classification, including epilepsy detection (2) compare the influence of the different frequency scales on the efficacy and diagnostic ability of the TE-FB-CEP EEG analysis. TE-FB-CEPs are used to take the advantage of the modulation energy tracking capability of the Teager energy operator (TEO). EEG signal is nonstationary in nature; it contains high frequency information with short interval segment and low frequency information with long period segment. Computation of conventional CEP demands the EEG segment to be stationary. Hence, the EEG segment length must be short enough to meet stationarity requirement, while long enough to capture specific patterns. TE-FB-CEPs computation, however, does not demand such requirements. In this study, we investigate and compare the Teager energy based linear, logarithmic, and Mel frequency, filter-bank cepstra. We also compare the performance of these methods in terms of their corresponding composite vectors in discriminating the eight classes on the general EEG database by Andrzejak et al. [16]. The performance of these methods is also compared with those of other researchers who had used the same database.

There are two variants in the approach adopted in automated detection of seizures. The first is based on a set of heuristic rules and thresholds. The second is based on classifier which employs pattern recognition techniques. In the former approach the results depend upon a single operating point and hence there is no much control over the accuracy. On the other hand, the latter permits the classifier to adapt to the desired performance and meet the requirements. Hence, we go in for the latter approach. From this perspective, an artificial neural network is well suited as a classifier. As such there is no well-established method to select an optimal network for classification. The rationale behind choosing radial basis function neural network (RBFNN) is that (1) the earlier literature shows that RBFNN is a more suitable classifier in medical applications because of its simplicity and faster learning abilities due to locally tuned neurons [17]; (2) RBFNN is also suitable from the point of view of its high speed, high accuracy, strong tolerance to input noise, and real-time property in updating network structure [17].

\section{Materials and Methods}

2.1. EEG Records. The EEG data used for this work is from University of Bonn EEG database which is available in public domain [16]. The choice of this database is based on the rationale that many seizure detection methods have employed this database and it becomes easy to compare the end results. The database consists of five sets (designated $\mathrm{Z}, \mathrm{O}$, N, F, and S) each containing 100 single channel EEG segments of 23.6-second duration. These segments have been picked from continuous multichannel EEG recordings after removal of any artifacts, like, muscle activity or eye movements, making sure that they fulfilled stationarity requirements. Sets $\mathrm{Z}$ and $\mathrm{O}$ contain segments taken from surface EEG recordings acquired from five healthy volunteers using a standard 1020 electrode placement scheme. The subjects were awake and relaxed with their eyes open for set $\mathrm{Z}$ and eyes closed for set $\mathrm{O}$, respectively. The segments for sets $\mathrm{N}, \mathrm{F}$, and $\mathrm{S}$ were acquired from five epileptic patients undergoing presurgical diagnosis. The type of epilepsy identified was temporal lobe epilepsy with the epileptogenic focus as the hippocampal formation. These recordings were taken from intracranial electrodes as they offer the most precise access to the emergence of seizures. Sets $\mathrm{N}$ and $\mathrm{F}$ contained only activity measured 
during seizure free intervals (interictal epileptiform activity), with segments in set $\mathrm{N}$ recorded from hippocampal formation of the opposite hemisphere of the brain and those in set $\mathrm{F}$ recorded within epileptogenic zone. On the other hand, set S contained only seizure activity (ictal intervals), with all segments recorded from sites exhibiting ictal activity. The patients had attained complete seizure control after resection of one of the hippocampal formations which was confirmed to be the epileptogenic zone. All the EEG signals were recorded using the same 128-channel amplifier system using an average common reference. The data were digitized at 173.61 samples per sec with 12 bit resolution. The bandpass filter setting was at $0.53-40 \mathrm{~Hz}(12 \mathrm{~dB} /$ octave $)$. Each single channel EEG segment has 4096 samples.

2.2. Teager Energy. The rationale of adopting TE-FB-CEP derived from nonlinear Teager energy (TE) in this study is based on the following reasons. (1) Seizures are abnormal sudden discharges in the brain which are represented in the EEG recordings by frequency changes and increased amplitude. The ability to accurately capture these changes is the key to detection of nonseizure and seizure states. TE is a feature very sensitive to variations of signal amplitude and frequency. (2) TE feature requires only four samples for its computation at any given instant and hence is computationally very efficient. (3) EEG signals are known to be nonstationary in nature [18]. We hypothesize that epochs of normal, interictal epileptic, and seizure belong to different nonlinear physiological processes. TE operator is a nonlinear operator which can be used for the estimation of energy of a nonstationary signal and hence TE is well suited for discriminating these episodes. Conventionally, CEP is derived from the original signal under investigation. However, the TECEP is derived from the TE of the signal, which is richer in information than the signal itself, as explained below.

If a signal sample is represented as $x_{n}=A \cos (\Omega n+\Phi)$, where $A$ is the amplitude and $\Phi$ is the initial phase. $\Omega$ is the digital frequency in radians/sample and is given by $\Omega=$ $2 \pi f / f_{s}$, where $f$ is the analog frequency in $\mathrm{Hz}$ and $f_{s}$ is the sampling frequency in $\mathrm{Hz}$. Then as per the TE algorithm the instantaneous TE, $E_{n}$ at a given instant of time $n$, is given by $[19,20]$

$$
\begin{aligned}
& E_{n}=x_{n}^{2}-x_{n-1} x_{n+1}=A^{2} \sin ^{2}(\Omega), \\
& E_{n} \approx A^{2} \Omega^{2}
\end{aligned}
$$

for small $\Omega$. With $\Omega<\pi / 4$ or $f / f_{s}<1 / 8$, the relative error in the last approximation is always less than $11 \%$. From (1) it is clear that the instantaneous TE can track modulation energy and identify instantaneous signal amplitude and also corresponding instantaneous frequency. For example, in a normal subject there is a fine balance in the brain between factors that generate electrical activity and factors that restrict it, and there are also systems that limit the spread of the electrical activity. Usually, during a seizure, these limits break down and an abnormal hypersynchronous neuronal activity due to a large number of neurons in the cerebral cortex of the brain occurs. The cerebral activity during an epileptic seizure is completely different from that of the interictal state or that of a normal subject. During interictal state the EEG is normal with occasional transient waveforms and apparently random with higher complexity, while during seizure the EEG tends to become hypersynchronized and cyclical with decreased complexity [21, 22]. Unlike the usual instantaneous signal energy which is only proportional to squared instantaneous amplitude, TE is proportional to the squared product of both instantaneous amplitude and instantaneous frequency. This new energy measure is therefore capable of responding rapidly to changes in both amplitude and frequency. Consequently disturbances in EEG signal generation and conduction path get reflected in the TEO energy $[23,24]$.

The general form of Teager nonlinear energy operator in the time domain for a discrete time signal $x[n]$ as given by Plotkin and Swamy [25-27] is

$$
\Psi_{\mathrm{td}}\{x[n]\}=x[n-l] x[n-m]-x[n-p] x[n-q],
$$

where $l+m=p+q$ and $\Psi_{\text {td }}$ denotes generalized TEO.

They showed that for $l \neq m$ and $p \neq q, \Psi_{\text {td }}$ is very robust to noise. That is, if the input signal contains additive white noise, then the output of (2) will not contain a component related to input noise. This is attributed to the removal of the square term in (2) while satisfying the conditions $l+m=p+q$, $l \neq m$, and $p \neq q$. In this work, we empirically found that the combination $l=1, m=2, p=0$, and $q=3$ is a suitable choice for noise reduction in EEG signals.

2.3. Cepstrum (CEP) Derived from Log Magnitude Spectrum. Cepstrum (CEP) analysis is a nonlinear signal processing technique with a variety of applications in areas such as speech and image processing. Among the speech recognition approaches the family based on CEP has been prominent due to its performance and simplicity. CEP models a time evolving signal as an ordered set of coefficients representing the signal spectral envelope. This in fact is a curve passing close to the peaks in the original spectrum. The CEP, though a compact representation, has been found to capture most of the relevant information in the original time series. It is possible to compare two relatively long time series with only a few cepstral coefficients. This implies that if two cepstral series are close then the corresponding signals have a similar evolution in time.

The real CEP is defined as the inverse Fourier transform of the log magnitude spectrum as given by

$$
C_{r}[k]=\operatorname{IDFT}\{\log |\mathrm{DFT}\{x[n]\}|\},
$$

where $C_{r}[k]$ represents $k$ th order real cepstral coefficient. If the inverse Fourier transform is replaced by discrete cosine transform (DCT), the resulting equation becomes

$$
C[k]=\operatorname{DCT}\{\log |\operatorname{DFT}\{x[n]\}|\},
$$

where $C[k]$ represents $k$ th order pseudocepstral coefficient.

The advantages are that (1) DCT has better energy compaction properties than the DFT and hence decreases memory requirements; (2) it reduces the computational complexity drastically without degrading the information 
content in the CEP and hence decreases execution time; and (3) DCT produces highly uncorrelated features. The resulting sequence of coefficients $C[k]$, called pseudo-CEP, is an approximation to the CEP, and in reality simply represents an orthogonal and compact representation of the log magnitude spectrum. The difference between cepstral coefficients of different time series can serve as a similarity measure among these time series. The cepstral coefficients decay rapidly to zero and hence, only the first few coefficients are needed to capture most of the dynamic information in the time series. This property of cepstral coefficients helps in reducing the dimensionality. Also, the number of coefficients to be retained does not depend upon the length of the time series. Moreover, the higher order coefficients represent the excitation process which is less useful. The coefficient $C[0]$ is similar to log energy (or DC component) of the signal and represents the segment energy. It is, usually, not treated as a cepstral coefficient and in this study, we drop $C[0]$.

\subsection{Teager Energy Based Filter-Bank Cepstra (TE-FB-CEPs).} As mentioned above, during interictal state the EEG is normal with occasional transient waveforms and apparently random with higher complexity, while during seizure the EEG tends to become hypersynchronized and cyclical with decreased complexity. These disturbances in EEG signal generation and conduction path manifest in the TE energy. We hypothesize that different states of EEG affect different frequency bands differently and an improved seizure classification features could be obtained by analyzing TE energy in different frequency bands. In this context, we also study the effect of linear, logarithmic, and Mel frequency filter-banks on the overall accuracy in general EEG data classification.

For a given EEG segment we compute the instantaneous TE based on (2). Treating this TEO output as signal, the TEO output is divided into a few subbands using a multirate filterbank. The filter-bank uses triangular filters spread over the whole frequency range from zero up to Nyquist frequency. The centre frequencies and the bandwidths are determined by the frequency scaling of the filter-bank. Depending on the frequency scaling used for filter-banks, we have three types of TE-FB-CEPs: (1) linear frequency scale TE-FB-CEP, (2) logarithmic frequency scale TE-FB-CEP, and (3) Mel frequency scale TE-FB-CEP. The magnitude spectrum of the TEO output is computed and warped to the frequency scale of the corresponding filter-bank followed by the usual log and DCT computation using (4) to obtain the TE-FB-CEP of the EEG segment under consideration. We designate the resulting cepstrum by $C_{T}[k]$. The coefficient $C_{T}[0]$ is similar to log energy (or DC component) of the TE signal. Unlike CEP, in this study, we account for $C_{T}[0]$. There are two important differences between TE-FB-CEPs and other Teager energy operator based cepstral features (TEOCEP, TECC, and T-MFCC): (1) in the computation of TE-FB-CEPs, TEO is first applied to raw signal and TEO output is fed to filter-bank. The cepstral coefficients are derived from filter-bank output. In the computation of the other Teager energy operator based cepstral features, the raw signal is applied to filter-bank. TEO is then applied to filter-bank output from which cepstral coefficients are derived; (2) computation of TE-FB-CEPs uses filter-bank with triangular filters, while the computation of latter uses mostly Gammatone filter-bank, which is suitable in speech processing.

EEG signals tend to be arbitrary in nature, and with some epileptic conditions, the frequency of the signal can change drastically with time depending upon the severity of the condition. In particular, during seizure the frequency components of the EEG signal become extremely erratic and unpredictable. To reduce the edge effects, a Hanning window was applied before spectrum was computed for such signals.

2.5. Radial Basis Function Neural Network (RBFNN). The recent research activities which use neural networks for classification have established that neural networks being powerful tools for pattern recognition problems can be a promising alternative to conventional methods of classification. The main advantage of neural networks lies in the fact that it makes use of self-adaptive techniques to adjust to the data without any explicit specification. In this work we employ radial basis function neural network (RBFNN) for the classification of normal, nonseizure, and seizure segments through TE-FB-CEPs derived from EEG signals. RBFNN has advantages of easy design, good generalization, strong tolerance to input noise, and online learning ability. The properties of RBF networks make it very suitable to design flexible control systems [17].

RBFNNs are nonlinear hybrid networks, which usually contain a single layer of hidden neurons. There are three layers: an input layer, a hidden layer, and an output layer. Each input neuron corresponds to an element from the input vector and is connected to the $k$ hidden layer neurons. Each hidden neuron is connected to the output neurons. The number of neurons in the output layer is equal to the number of possible classes $n$ in the classification problem. The input layer broadcasts the coordinates of the input vector to each of the nodes in the hidden layer. Each node in the hidden layer then produces an activation based on the associated radial basis function. Finally, each node in the output layer computes a linear combination of the activations from the hidden nodes. The output nodes from a RBFNN can be described as

$$
C_{j}(x)=\sum_{i} w_{j i}\left\|x-\mu_{i}\right\| \sigma_{i} \quad 1 \leq i \leq k, 1 \leq j \leq n,
$$

where $C_{j}(x)$ represents the function corresponding to the $j$ th output unit or class- $j$ and is a linear combination of $k$ radial basis functions with center $\mu_{i}$ and bandwidth $\sigma_{i} . w_{j}$ is the weight vector of class- $j$ and $w_{j i}$ is the weight of $j$ th class and $i$ th center. The commonly used basis function in the RBFNN to solve pattern recognition problems is a Gaussian function and with this (5) becomes

$$
C_{j}(x)=\sum_{i} w_{j i} \exp \left(\frac{\left\|x-\mu_{i}\right\|^{2}}{\left(2 \sigma_{i}^{2}\right)}\right) \quad 1 \leq i \leq k, 1 \leq j \leq n .
$$

From (6) it can be observed that the output of RBFNN depends upon total number of neurons $k$, the weights 
between the output and the hidden layer $w_{j i}$, the centers of neurons $\mu_{i}$, and the bandwidths of the neurons $\sigma_{i}$. This implies that the performance of RBFNN is determined by the selection of the right parameters. RBFNN can be trained in different ways. In one of the conventional methods, the training begins with a predetermined network structure. Then the centers and the bandwidths are trained. Again, several methods are proposed to find the centers of which clustering based methods are popular.

In this work, we use MATLAB toolbox which greatly simplifies the implementation of the required RBFNN. The function newrb is used to approximate functions/vectors defined by a set of data points. Newrb adds neurons to the hidden layer of a radial basis network until it meets the specified mean squared error goal. The function newrb uses a radial basis layer which requires a parameter, spread (related to bandwidth of the neuron), to be fixed. It is important that the spread constant be large enough that the radial basis layer neurons respond to overlapping regions of the input space, but not so large that all the neurons respond in essentially the same manner. In other words, the parameter spread decides the fitment to the function. The larger the spread, the smoother the function approximation. Too large spread means that a lot of neurons are required to fit a fast-changing function. Too small spread means many that neurons are required to fit a smooth function, and the network might not generalize well. The function newrb is called with different spreads to find the best value for a given problem. A simple thumb rule is to choose a spread constant larger than the distance between adjacent input vectors, so as to get good generalization, but smaller than the distance across the whole input space.

\section{Results and Discussion}

In this work, we handle all the different classification problems proposed by Guo et al. [28] and Tzallas et al. [29, 30] to encompass all the possible discriminations in the medical field related to epilepsy and compare the performance of our approach with those of other researchers.

(1) In the first classification problem, two classes are examined, normal and seizure. The normal class includes only set $Z$ while seizure class includes set $S$. In this classification problem, $200 \mathrm{EEG}$ segments are included.

(2) In the second classification two classes, namely, nonseizure and seizure are examined, but not all sets are used. The nonseizure class includes sets $\mathrm{Z}, \mathrm{N}$, and F while seizure class includes set $\mathrm{S}$. In this classification problem, the dataset includes $400 \mathrm{EEG}$ segments.

(3) In the third problem, again, two classes, nonseizure and seizure are examined. Now the nonseizure class includes sets $\mathrm{Z}, \mathrm{O}, \mathrm{N}$, and $\mathrm{F}$ while seizure class includes set $\mathrm{S}$. In this classification problem, $500 \mathrm{EEG}$ segments are included in the dataset.

(4) In the fourth classification problem, three classes are examined, normal, nonseizure, and seizure, but not all sets are used. The normal class includes only set $\mathrm{Z}$, nonseizure class includes set $\mathrm{F}$, while seizure class includes set $S$. In this case, 300 EEG segments are used.

(5) The fifth classification problem takes care of five datasets comprising 500 EEG segments into three classes, normal ( $\mathrm{Z}$ and $\mathrm{O})$, nonseizure $(\mathrm{N}$ and $\mathrm{F})$, and seizure (S).

(6) The sixth classification problem handles five datasets comprising $500 \mathrm{EEG}$ segments into five individual classes, eyes-open $(\mathrm{Z})$, eyes-closed $(\mathrm{O})$, nonseizure interictal $(\mathrm{N})$, nonseizure interictal $(\mathrm{F})$, and seizure (S).

(7) In the seventh classification problem, three datasets comprising 300 EEG segments into two classes, nonseizure ( $\mathrm{N}$ and $\mathrm{F}$ ) and seizure (S) are examined.

(8) Finally, in the eighth classification problem, three classes are examined, normal, nonseizure, and seizure, but not all sets are used. The normal class includes only set Z; nonseizure class includes set $\mathrm{N}$, while seizure class includes set $\mathrm{S}$. In this case, 300 EEG segments are used.

The first three classification problems were proposed by Guo et al. [28], the next three classification problems were proposed by Tzallas et al. $[29,30]$, while the seventh and eighth are proposed by us. These classification problems have been chosen such that they are close to clinical applications.

Now, we compare the diagnostic capability of the three TE-FB-CEPs: (1) linear scale TE-FB-CEP, (2) logarithmic scale TE-FB-CEP, and (3) Mel scale TE-FB-CEP and their composite vectors in the above eight classification problems using RBFNN. Empirically, we had found that repeating the same procedure as in [11], an analysis window length, $W \geq$ 868 samples (5.0 seconds), a spread constant, $s \leq 2$ for RBFNN, and a number of cepstral coefficients, $N \geq 9$ leads to optimum results in all the eight cases. In this work, an 868-sample sliding window with $50 \%$ overlap between consecutive windows, $N=9$ and $s=2$, is used in the computation of TE-FB-CEP. Distance-based classifiers demand normalization of the data and hence feature vectors are normalized before they are applied to RBFNN.

First we compare the results of the performance of the three TE-FB-CEP baseline vectors in the general EEG seizure detection. The comparison is tried on each of the above mentioned eight different classification problems which have been widely used in the literature related to epilepsy. Typical EEG segments, one from each dataset (in the order Z, O, N, F, and $S$ ), are shown in Figure 1. We adopted leave-one-recordout cross-validation scheme. In specific, we run 10 runs of a 10 -fold cross-validation (with 10 runs for each fold split), thus having a total of 100 RBFNN runs to average to produce the final result. With each new fold split, the EEG data segments are randomized. Descriptive results of RBFNN analysis using TE-FB-CEP baseline vectors for discriminating different classification problems are depicted in Table 1. It is found that all the three TE-FB-CEP baseline feature vectors exhibit excellent performance, with logarithmic frequency scaling 
TABLE 1: Percentage average accuracy of RBFNN analysis using linear, logarithmic, and Mel scale TE-FB-CEP methods $(W=868, N=9$, and $s=2$ ) for baseline vectors in discriminating eight classification problems (CPs).

\begin{tabular}{lccc}
\hline CP & Linear scale TE-FB-CEP & Logarithmic scale TE-FB-CEP & Mel scale TE-FB-CEP \\
\hline 1 & 81.65 & 82.37 & 81.60 \\
2 & 89.05 & 90.36 & 88.98 \\
3 & 92.38 & 93.07 & 91.61 \\
4 & 82.08 & 82.89 & 81.72 \\
5 & 81.84 & 82.78 & 81.53 \\
6 & 81.38 & 81.50 & 79.50 \\
7 & 85.31 & 86.04 & 85.30 \\
8 & 84.07 & 84.09 & 83.42 \\
\hline
\end{tabular}

TABLE 2: Percentage average accuracy of RBFNN analysis using linear, logarithmic, and Mel scale TE-FB-CEP methods $(W=868, N=9$, and $s=2$ ) for composite vector-1 in discriminating eight classification problems (CPs).

\begin{tabular}{lccc}
\hline CP & Linear scale TE-FB-CEP & Logarithmic scale TE-FB-CEP & Mel scale TE-FB-CEP \\
\hline 1 & 97.40 & 97.93 & 98.09 \\
2 & 96.37 & 97.05 & 96.13 \\
3 & 95.31 & 96.92 & 95.29 \\
4 & 98.57 & 98.89 & 98.55 \\
5 & 96.50 & 98.11 & 96.27 \\
6 & 97.18 & 98.01 & 96.88 \\
7 & 97.08 & 98.09 & 97.63 \\
8 & 98.73 & 98.94 & 98.65 \\
\hline
\end{tabular}
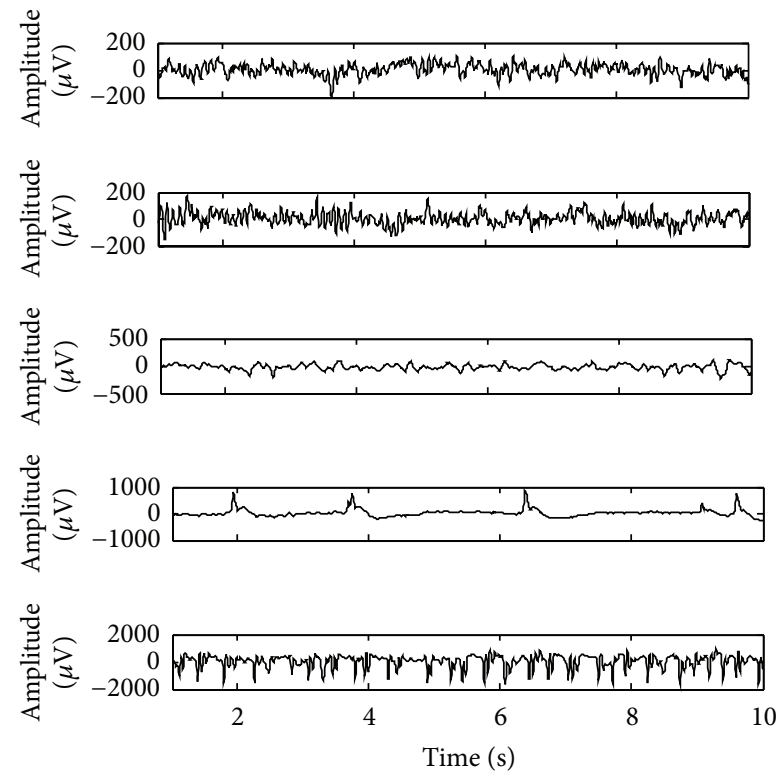

FIgUre 1: Typical EEG segments from each of the five sets $(\mathrm{Z}, \mathrm{O}, \mathrm{N}$, F, and S), from top to bottom.

showing the best performance among the three cases, in all the CPs.

The results of RBFNN analysis using composite cepstral vectors for discriminating different classification problems in the three TE-FB-CEP methods are shown in Tables 2 and 3 . The first composite vector includes velocity vector together with the static cepstral vector. The second composite vector includes velocity and acceleration vectors together with the static cepstral vector. It is found that the first and second composite vectors with logarithmic frequency scaling outperform in all the CPs. Nevertheless, the composite cepstral vectors, with linear and Mel frequency scaling methods too, show an excellent performance. This is in agreement with applications in other domains of signal processing where the composite vectors, in general, enhance the performance. For example, the composite cepstral vectors of TEOCEP and TECC add to improved performance in speech processing $[12,14]$. In a recent study [11], on the other hand, we had found that the overall performance of both the composite vectors of the traditional cepstrum (CEP) deteriorated compared to that of the baseline vector in the seizure detection and classification of EEG segments. It was interesting to note that the baseline CEP vector alone showed the best performance. The composite CEP vectors, instead of at least maintaining best performance, showed a degraded performance. This implied that the velocity and acceleration CEP features were hurting the performance, probably because of the nonlinearities introduced in the EEG significantly affected the computation of derivatives. However, in contrast to this behavior, the composite vectors in all the three TE-FB-CEP methods, irrespective of the frequency scale (linear, log, or Mel), maintain excellent overall accuracy in all the eight classification problems which are close to clinical applications.

Various researchers have proposed different methods for epileptic seizure detection using the database by Andrzejak et al. [16]. Table 4 provides a comparison between 
TABle 3: Percentage average accuracy of RBFNN analysis using linear, logarithmic, and Mel scale TE-FB-CEP methods $(W=868, N=9$, and $s=2$ ) for composite vector-2 in discriminating eight classification problems (CPs).

\begin{tabular}{lccc}
\hline CP & Linear scale TE-FB-CEP & Logarithmic scale TE-FB-CEP & Mel scale TE-FB-CEP \\
\hline 1 & 98.51 & 99.99 & 98.40 \\
2 & 97.05 & 98.90 & 97.08 \\
3 & 97.20 & 98.73 & 97.29 \\
4 & 98.62 & 99.28 & 98.72 \\
5 & 98.26 & 98.72 & 98.41 \\
6 & 99.22 & 99.30 & 99.20 \\
7 & 97.88 & 98.61 & 98.12 \\
8 & 98.83 & 99.12 & 98.76 \\
\hline
\end{tabular}

TABLE 4: A comparison of classification accuracy achieved by our method and best performed others' method for eight classification problems. $\mathrm{CP}$ stands for classification problem.

\begin{tabular}{|c|c|c|c|c|c|}
\hline $\begin{array}{l}\text { CP } \\
\text { No. }\end{array}$ & $\begin{array}{l}\text { Number } \\
\text { of classes }\end{array}$ & $\begin{array}{l}\text { Researcher } \\
\text { (year) }\end{array}$ & Method & Dataset & $\begin{array}{l}\text { Classification } \\
\text { accuracy }(\%)\end{array}$ \\
\hline 1 & 2 & Tzallas et al., (2007) $[29,30]$ & Time-frequency analysis and ANN & $\mathrm{Z}, \mathrm{S}$ & 100.0 \\
\hline 1 & 2 & $\begin{array}{l}\text { Subasi and Gursoy (2010) } \\
{[31]}\end{array}$ & $\begin{array}{l}\text { Principal component analysis, independent component } \\
\text { analysis, linear discriminant analysis, and support } \\
\text { vector machines }\end{array}$ & $\mathrm{Z}, \mathrm{S}$ & 100.0 \\
\hline 1 & 2 & Guo et al., (2010) [28] & $\begin{array}{l}\text { Discrete wavelet transform, line length feature, and } \\
\text { MLPNN }\end{array}$ & $\mathrm{Z}, \mathrm{S}$ & 99.6 \\
\hline 1 & 2 & Guo et al., (2011) [32] & $\begin{array}{l}\text { Genetic programming based feature extraction and } \\
\text { k-nearest neighbors classifier }\end{array}$ & $\mathrm{Z}, \mathrm{S}$ & 99.0 \\
\hline 1 & 2 & Wang et al., (2011) [33] & Wavelet transform and Shannon entropy & $\mathrm{Z}, \mathrm{S}$ & 100.0 \\
\hline 1 & 2 & Iscan et al., (2011) [34] & $\begin{array}{l}\text { Cross-correlation, power spectral density, support } \\
\text { vector machines, linear discriminant analysis, and } \\
k \text {-nearest neighbors classifier }\end{array}$ & $\mathrm{Z}, \mathrm{S}$ & 100.0 \\
\hline 1 & 2 & Orhan et al., (2011) [35] & $\begin{array}{l}\text { Wavelet transform, k-nearest neighbors classifier, and } \\
\text { ANN }\end{array}$ & $\mathrm{Z}, \mathrm{S}$ & 100.0 \\
\hline 1 & 2 & This work (2013) & TE-FB-CEP feature vectors and RBFNN & $\mathrm{Z}, \mathrm{S}$ & 99.99 \\
\hline 2 & 2 & Ocak (2009) [36] & Discrete wavelet transform and approximate entropy & ZNF, S & 96.65 \\
\hline 2 & 2 & Guo et al., (2010) [28] & $\begin{array}{l}\text { Discrete wavelet transform, line length feature and } \\
\text { MLPNN }\end{array}$ & ZNF, S & 97.75 \\
\hline 2 & 2 & This work (2013) & TE-FB-CEP feature vectors and RBFNN & ZNF, S & 97.90 \\
\hline 3 & 2 & Tzallas et al., (2007) $[29,30]$ & Time-frequency analysis and ANN & ZONF, S & 97.73 \\
\hline 3 & 2 & Guo et al., (2010) [28] & $\begin{array}{l}\text { Discrete wavelet transform, line length feature, and } \\
\text { MLPNN }\end{array}$ & ZONF, S & 97.77 \\
\hline 3 & 2 & Orhan et al., (2011) [35] & $\begin{array}{l}\text { Wavelet transform, k-nearest neighbors classifier, and } \\
\text { ANN }\end{array}$ & ZONF, S & 100.0 \\
\hline 3 & 2 & This work (2013) & TE-FB-CEP feature vectors and RBFNN & ZONF, S & 98.73 \\
\hline 4 & 3 & Güler and Übeyli (2005) [37] & $\begin{array}{l}\text { Lyapunov exponents, recurrent neural } \\
\text { network (RNN) }\end{array}$ & Z, F, S & 96.79 \\
\hline 4 & 3 & Tzallas et al., (2007) $[29,30]$ & Time-frequency analysis and ANN & Z, F, S & 99.28 \\
\hline 4 & 3 & This work (2013) & TE-FB-CEP feature vectors and RBFNN & $\mathrm{Z}, \mathrm{F}, \mathrm{S}$ & 99.28 \\
\hline 5 & 3 & Guo et al., (2010) [28] & Wavelet transform, line length, and ANN & $\mathrm{ZO}, \mathrm{NF}, \mathrm{S}$ & 97.77 \\
\hline 5 & 3 & Orhan et al., (2011) [35] & $\begin{array}{l}\text { Wavelet transform, k-nearest neighbors classifier, and } \\
\text { ANN }\end{array}$ & $\mathrm{ZO}, \mathrm{NF}, \mathrm{S}$ & 95.60 \\
\hline 5 & 3 & This work (2013) & TE-FB-CEP feature vectors and RBFNN & $\mathrm{ZO}, \mathrm{NF}, \mathrm{S}$ & 98.72 \\
\hline 6 & 5 & Güler and Übeyli (2007) [37] & $\begin{array}{l}\text { Wavelet transform, Lyapunov exponents-support vector } \\
\text { machine }\end{array}$ & $\mathrm{Z}, \mathrm{O}, \mathrm{N}, \mathrm{F}, \mathrm{S}$ & 99.28 \\
\hline 6 & 5 & Übeyli (2010) [38] & Lyapunov exponents and PNN & $\mathrm{Z}, \mathrm{O}, \mathrm{N}, \mathrm{F}, \mathrm{S}$ & 98.05 \\
\hline 6 & 5 & This work (2013) & TE-FB-CEP feature vectors and RBFNN & $\mathrm{Z}, \mathrm{O}, \mathrm{N}, \mathrm{F}, \mathrm{S}$ & 99.30 \\
\hline 7 & 2 & This work (2013) & TE-FB-CEP feature vectors and RBFNN & NF, $S$ & 99.12 \\
\hline
\end{tabular}


our method (using a logarithmic frequency scaling) and other methods that have used the same database. In the table, we present a listing of the method, dataset used, and classification accuracy, for the eight classification problems. It is to be noted that all the methods shown in the table, including ours, had used modern classifiers for first training and then classification. In the first classification problem (CP no. 1), the results obtained by Tzallas et al. [29, 30], Subasi and Gursoy [31], Wang et al. [33], Iscan et al. [34], and Orhan et al. [35] are the best (100\%). Our method yielded an accuracy of $99.99 \%$ which is almost $100 \%$. In the second problem (CP no. 2), our method shows the best results (98.90\%). For the third classification problem (CP no. 3), the result found by Orhan et al. [35] is the best (100\%). Our method showed an average accuracy of $98.73 \%$, which is better than those by Tzallas et al. [30] and Guo et al. [28]. It is found that for the fourth classification problem (CP no. 4), our method works on par with that of Tzallas et al. [30], each with an overall accuracy equal to $99.28 \%$. For the fifth and sixth classification problems (CP nos. 5 and 6), our method showed the highest average accuracy. In the seventh and eighth classification problems (CP nos. 7 and 8), the new classification problems appended by us in this paper, the results are found to be excellent. All these results collectively show a considerable improvement in our approach over many of the previous epilepsy detection methods. The above comparison also implies that an automated system developed based on this approach should provide feedback to the experts for quick and accurate EEG classification.

The database used has already been preprocessed by the removal of artifacts by visual inspection. This is a limitation of our method (like many who have used the same database). Nevertheless, the results of this study provide sufficient evidence to warrant the assessment under actual clinical situations that can provide more robust confirmation of the application of this approach to capture diagnostically significant information. Hence the method is well suited for implementation not only in epilepsy detection system, but also in applications, such as seizure warning systems, closed loop seizure control systems, or delivering abortive responses/monitoring patients using implantable therapeutic devices [39].

\section{Conclusions}

A comparison of the EEG epileptic seizure detection and classification based on baseline and composite vectors comprising velocity and acceleration features, using TE-FB-CEP methods, is presented. In the literature it is found that in the applications, such as speech analysis and recognition the velocity and acceleration features do enhance the performance. However, our previous study showed that in the case of EEG discrimination using CEP method, the velocity and acceleration features were hurting the performance. The chief finding of this study is that unlike CEP method, in the TE-FBCEP methods, irrespective of the frequency scale employed the composite vectors exhibit excellent performance in the discrimination of EEG segments in a variety of classification problems close to clinical applications. Automated systems developed based on TE-FB-CEP methods should provide feedback to the clinical neurophysiologists for quick and accurate EEG discrimination. Such discrimination is important in some applications, such as seizure warning systems, closed loop seizure control systems, or delivering abortive responses/monitoring patients using implantable therapeutic devices.

\section{Conflict of Interests}

The author declares that he has no conflict of interests.

\section{Authors' Contribution}

The author, who is also the corresponding author, is the sole contributor to this work.

\section{References}

[1] K. Lehnertz, F. Mormann, T. Kreuz et al., "Seizure prediction by nonlinear EEG analysis," IEEE Engineering in Medicine and Biology Magazine, vol. 22, no. 1, pp. 57-63, 2003.

[2] F. Lopes Da Silva, W. Blanes, S. N. Kalitzin, J. Parra, P. Suffczynski, and D. N. Velis, "Epilepsies as dynamical diseases of brain systems: basic models of the transition between normal and epileptic activity," Epilepsia, vol. 44, no. 12, pp. 72-83, 2003.

[3] R. Flink, B. Pedersen, A. B. Guekht et al., "Guidelines for the use of EEG methodology in the diagnosis of epilepsy. International League Against Epilepsy: commission report. Commission on European Affairs: subcommission on European guidelines," Acta Neurologica Scandinavica, vol. 106, no. 1, pp. 1-7, 2002.

[4] N. Mc Grogan, Neural network detection of epileptic seizures in the electroencephalogram [Ph.D. thesis], Oxford University, Oxford, UK, 1999.

[5] A. Aarabi, R. Grebe, and F. Wallois, "A multistage knowledgebased system for EEG seizure detection in newborn infants," Clinical Neurophysiology, vol. 118, no. 12, pp. 2781-2797, 2007.

[6] B. R. Greene, S. Faul, W. P. Marnane, G. Lightbody, I. Korotchikova, and G. B. Boylan, "A comparison of quantitative EEG features for neonatal seizure detection," Clinical Neurophysiology, vol. 119, no. 6, pp. 1248-1261, 2008.

[7] A. Temko, E. Thomas, G. Boylan, W. Marnane, and G. Lightbody, "An SVM-based system and its performance for detection of seizures in neonates," in Proceedings of the 31st Annual International Conference of the IEEE Engineering in Medicine and Biology Society: Engineering the Future of Biomedicine (EMBC '09), pp. 2643-2646, September 2009.

[8] A. H. Shoeb, Application of machine learning to epileptic seizure onset detection and treatment [Ph.D. thesis], Massachusetts Institute of Technology, 2009.

[9] A. T. Tzallas, M. G. Tsipouras, and D. I. Fotiadis, "Epileptic seizure detection in EEGs using time-frequency analysis," IEEE Transactions on Information Technology in Biomedicine, vol. 13, no. 5, pp. 703-710, 2009.

[10] A. T. Tzallas, M. G. Tsipouras, D. G. Tsalikakis et al., "Automated epileptic seizure detection methods: a review study," in Epilepsy-Histological, Electroencephalographic and Psychological Aspects, 2012.

[11] C. Kamath, "Comparison of baseline cepstral vector and composite vectors in the automatic seizure detection using 
probabilistic neural networks," ISRN Biomedical Engineering, vol. 2013, Article ID 984864, 9 pages, 2013.

[12] F. Jabloun, A. E. Çetin, and E. Erzin, "Teager energy based feature parameters for speech recognition in car noise," IEEE Signal Processing Letters, vol. 6, no. 10, pp. 259-261, 1999.

[13] D. Dimitriadis, P. Maragos, and A. Potamianos, "Auditory teager energy cepstrum coefficients for robust speech recognition," in Proceedings of the 9th European Conference on Speech Communication and Technology, pp. 3013-3016, September 2005.

[14] P. Heracleous, "Using Teager energy cepstrum and HMM distances in automatic speech recognition and analysis of unvoiced speech," International Journal of Information and Communication Engineering, vol. 5, no. 1, pp. 31-37, 2009.

[15] H. A. Patil and T. K. Basu, "Identifying perceptually similar languages using Teager energy based cepstrum," Engineering Letters, vol. 16, no. 1, pp. 151-159, 2008.

[16] R. G. Andrzejak, K. Lehnertz, F. Mormann, C. Rieke, P. David, and C. E. Elger, "Indications of nonlinear deterministic and finite-dimensional structures in time series of brain electrical activity: dependence on recording region and brain state," Physical Review E, vol. 64, Article ID 061907, 2001.

[17] H. Yu, T. Xie, S. Paszczyñski, and B. M. Wilamowski, "Advantages of radial basis function networks for dynamic system design," IEEE Transactions on Industrial Electronics, vol. 58, no. 12, pp. 5438-5450, 2011.

[18] B. Litt, R. Esteller, J. Echauz et al., "Epileptic seizures may begin hours in advance of clinical onset: a report of five patients," Neuron, vol. 30, no. 1, pp. 51-64, 2001.

[19] T. F. Quatieri, Discrete-Time Speech Signal Processing, Principles and Practice, Pearson Education, Singapore, 2004.

[20] J. F. Kaiser, "On a simple algorithm to calculate the 'energy' of a signal," in Proceedings of the International Conference on Acoustics, Speech, and Signal Processing (ICASSP '90), pp. 381384, April 1990.

[21] A. Aarabi, F. Wallois, and R. Grebe, "Does spatiotemporal synchronization of EEG change prior to absence seizures?" Brain Research, vol. 1188, no. 1, pp. 207-221, 2008.

[22] W. Klonowski, "Everything you wanted to ask about EEG but were afraid to get the right answer," Nonlinear Biomedical Physics, vol. 3, article 2, 2009.

[23] C. Kamath, "A new approach to detect congestive heart failure using Teager energy nonlinear scatter plot of RR interval series," Medical Engineering and Physics, vol. 34, no. 7, pp. 841-848, 2012.

[24] C. Kamath, "ECG beat classification using features extracted from Teager energy functions in time and frequency domains," IET Signal Processing, vol. 5, no. 6, pp. 575-581, 2011.

[25] E. I. Plotkin and M. N. S. Swamy, "Multistage implementation of parameter-invariant null filter and its application to discrimination of closely spaced sinusoids," in Proceedings of IEEE International Symposium on Circuits and Systems, vol. 1, pp. 767770, 1988.

[26] E. I. Plotkin and M. N. S. Swamy, "Parameter-free structural modeling: a contribution to the solution of the separation of highly correlated AR-signals," in Proceedings of the IEEE International Symposium on Circuits and Systems (ISCAS '98), vol. 5, pp. 1-4, June 1998.

[27] E. I. Plotkin and M. N. S. Swamy, "Signal processing based on parameter structural modeling and separation of highly correlated signals of known structure," Circuits, Systems, and Signal Processing, vol. 17, no. 1, pp. 51-68, 1998.
[28] L. Guo, D. Rivero, J. Dorado, J. R. Rabuñal, and A. Pazos, “Automatic epileptic seizure detection in EEGs based on line length feature and artificial neural networks," Journal of Neuroscience Methods, vol. 191, no. 1, pp. 101-109, 2010.

[29] A. T. Tzallas, M. G. Tsipouras, and D. I. Fotiadis, "The use of time-frequency distributions for epileptic seizure detection in EEG recordings," in Proceedings of the Annual International Conference of the IEEE Engineering in Medicine and Biology Society, pp. 3-6, 2007.

[30] A. T. Tzallas, M. G. Tsipouras, and D. I. Fotiadis, "Automatic seizure detection based on time-frequency analysis and artificial neural networks," Computational Intelligence and Neuroscience, vol. 2007, Article ID 80510, 13 pages, 2007.

[31] A. Subasi and M. I. Gursoy, "EEG signal classification using PCA, ICA, LDA and support vector machines," Expert Systems with Applications, vol. 37, no. 12, pp. 8659-8666, 2010.

[32] L. Guo, D. Rivero, J. Dorado, C. R. Munteanu, and A. Pazos, "Automatic feature extraction using genetic programming: an application to epileptic EEG classification," Expert Systems with Applications, vol. 38, no. 8, pp. 10425-10436, 2011.

[33] D. Wang, D. Miao, and C. Xie, "Best basis-based wavelet packet entropy feature extraction and hierarchical EEG classification for epileptic detection," Expert Systems with Applications, vol. 38, no. 11, pp. 14314-14320, 2011.

[34] Z. Iscan, Z. Dokur, and T. Demiralp, "Classification of electroencephalogram signals with combined time and frequency features," Expert Systems with Applications, vol. 38, no. 8, pp. 10499-10505, 2011.

[35] U. Orhan, M. Hekim, and M. Ozer, "EEG signals classification using the K-means clustering and a multilayer perceptron neural network model," Expert Systems with Applications, vol. 38, no. 10, pp. 13475-13481, 2011.

[36] H. Ocak, "Automatic detection of epileptic seizures in EEG using discrete wavelet transform and approximate entropy," Expert Systems with Applications, vol. 36, no. 2, pp. 2027-2036, 2009.

[37] I. Güler and E. D. Übeyli, "Multiclass support vector machines for EEG-signals classification," IEEE Transactions on Information Technology in Biomedicine, vol. 11, no. 2, pp. 117-126, 2007.

[38] E. D. Übeyli, "Lyapunov exponents/probabilistic neural networks for analysis of EEG signals," Expert Systems with Applications, vol. 37, no. 2, pp. 985-992, 2010.

[39] S.-F. Liang, H.-C. Wang, and W.-L. Chang, "Combination of EEG complexity and spectral analysis for epilepsy diagnosis and seizure detection," Eurasip Journal on Advances in Signal Processing, vol. 2010, Article ID 853434, 15 pages, 2010. 

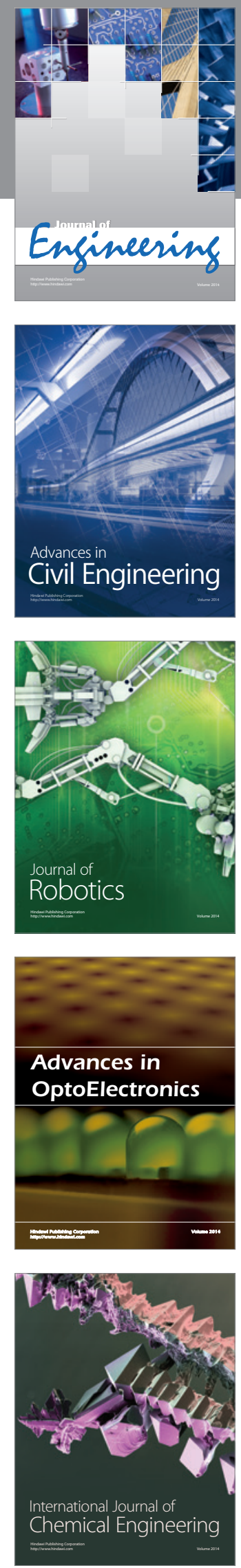

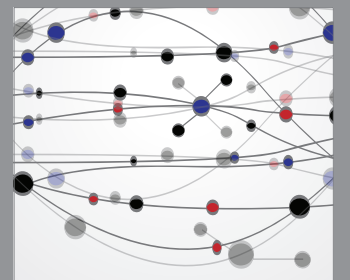

The Scientific World Journal
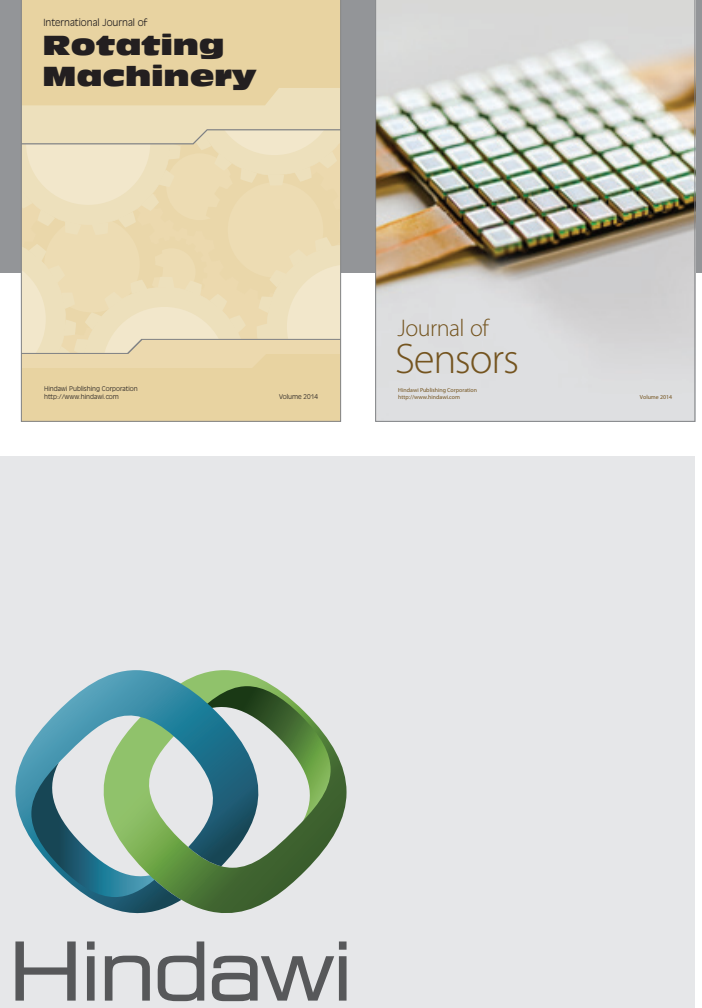

Submit your manuscripts at http://www.hindawi.com
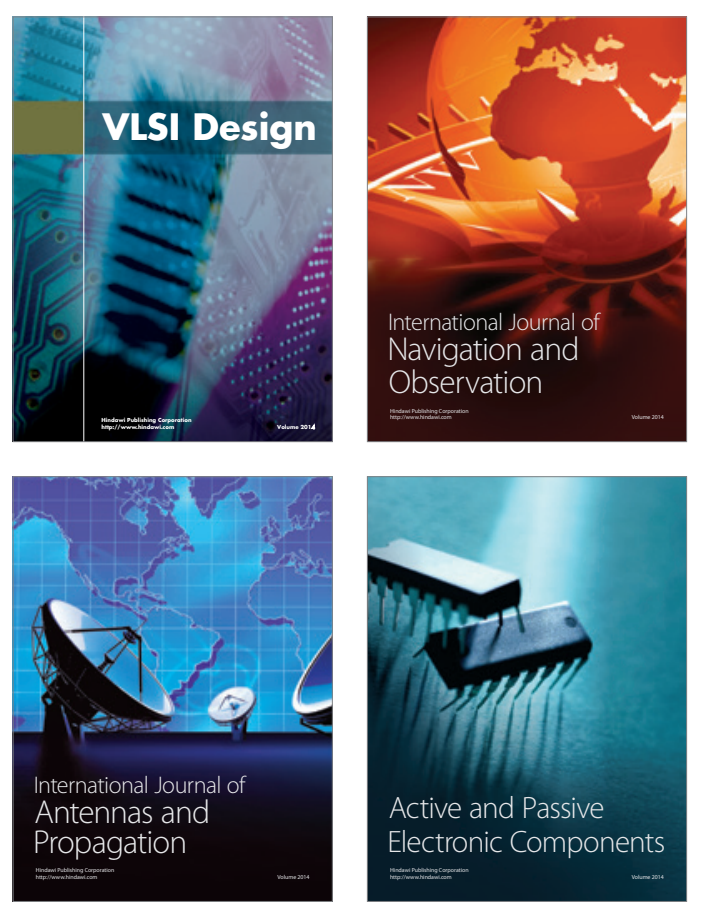
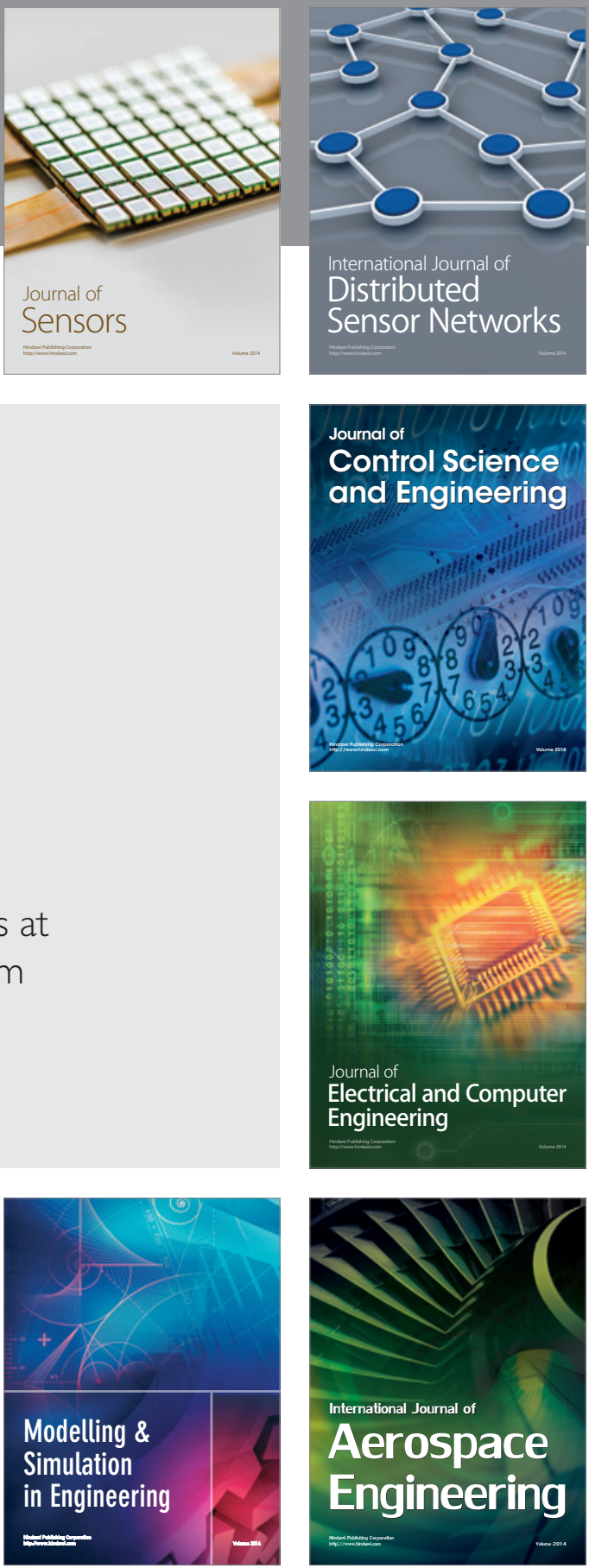

Journal of

Control Science

and Engineering
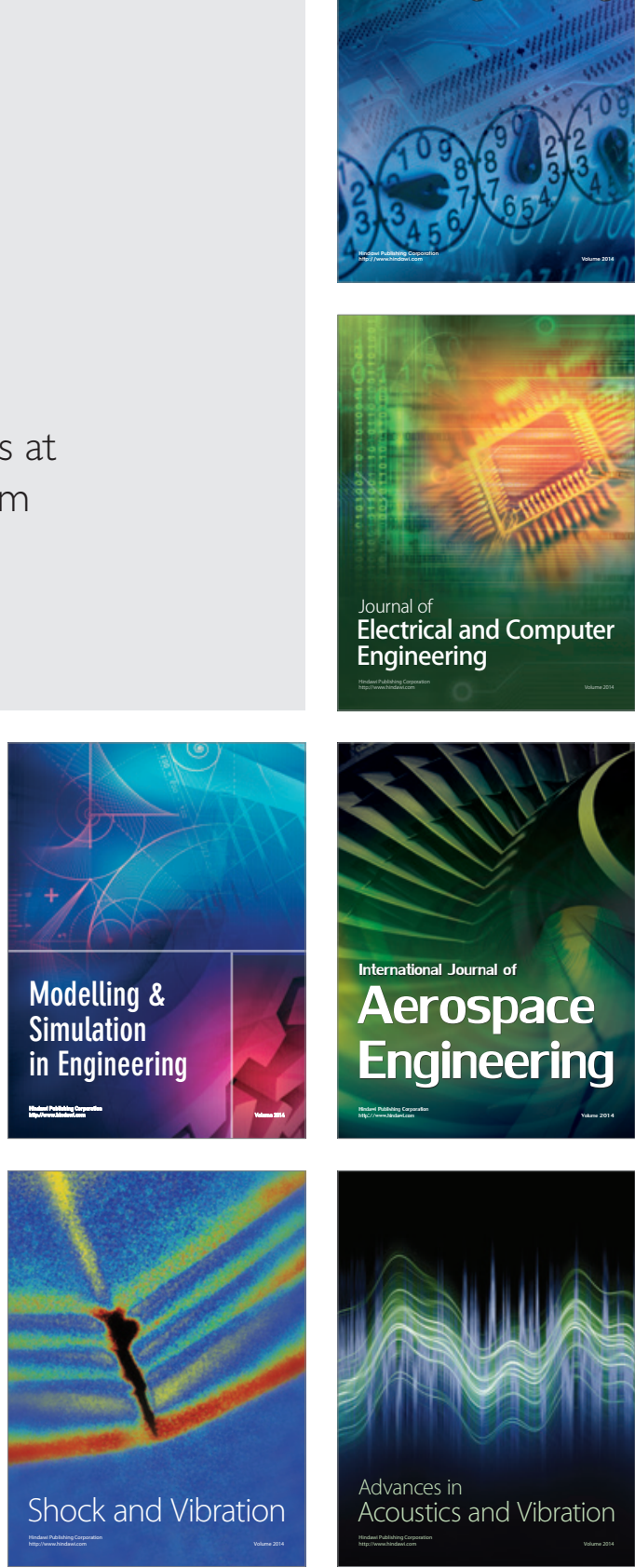